\title{
Synthesis of a Molecularly Imprinted Polymer for Dioxin
}

\section{Cosimino Malitesta ${ }^{1, *}$, Rosaria Anna Picca ${ }^{1}$, Giuseppe Ciccarella ${ }^{2}$, Vito Sgobba ${ }^{2}$ and Magda Brattoli $^{1}$}

1 Laboratorio di Chimica Analitica, Dipartimento di Scienza dei Materiali, Università degli Studi di Lecce, Palazzina M, Via Monteroni, 73100 Lecce, Italy

2 Dipartimento di Ingegneria dell’Innovazione, Università degli Studi di Lecce, Via Arnesano, 73100 Lecce, Italy

* Author to whom correspondence should be addressed; E-mail: cosimino.malitesta@unile.it

Received: 28 April 2006 / Accepted: 28 June 2006 / Published: 24 August 2006

\begin{abstract}
A molecularly imprinted polymer for recognising selectively 2,3,7,8tetrachlorodibenzodioxin (TCDD) was made by a new non-covalent method employing a "dummy" template. The proposed way represents a simplification of a synthetic scheme proposed by Lübke et al.[1] for covalent imprinting. Comparison of extraction yields of the novel polymer, a non imprinted polymer and an imprinting polymer, prepared by the original procedure demonstrates the binding capacity of the proposed polymer, which is in principle applicable to solid phase extraction (SPE) of dioxin.
\end{abstract}

Keywords: Molecularly imprinted polymers, non-covalent approach, 2,3,7,8tetrachlorodibenzodioxin.

\section{Introduction}

Determination of pollutants at ultra-trace levels requires often several pretreatment steps, comprising preconcentration operation. Classical separation techniques are based on liquid-liquid extraction. In the past years, Solid Phase Extraction (SPE) arose as a powerful alternative [2-4] to classical methods, as it is less time-consuming and more effective. This technique is based on selective adsorption of analytes at solid-liquid interface. In principle, selectivity can be improved by using a solid phase possessing molecular recognition capability. In particular, molecularly imprinted polymers (MIPs) represent very promising materials in this field [5-7]. In fact, molecular imprinting leads to the formation of binding sites for a target molecule (analyte) in the supramolecular architecture of polymer. During polymerisation, analyte, able to interact with monomer, acts as a template and, after 
its removal, high affinity sites are left into the cross-linked porous polymer. Such polymers show recognition properties similar to biological receptors but they are more stable and less expensive. Two distinct methodologies have been employed [8]: covalent and non-covalent imprinting, depending on the nature of interactions between template and monomer. Non-covalent imprinting, based on hydrogen bonding, Van der Waals forces, electrostatic or hydrophobic interactions, seems more versatile and easier than the former and it is the most employed for preparing SPE phases [9]. However, a key disadvantage of non-covalent approach is template bleeding, producing inaccurate results in trace analysis, caused by the possible template remained in the polymer after removal step.

Polychlorinated dibenzo-p-dioxins (PCDDs) represent toxic compounds whose determination is mandatory in several countries. PCDDs are by-products of waste incineration, fuel combustion and chlorine use in wood industry; they are classified as Persistent Organic Pollutants [10], characterised by high chemical stability, bioaccumulation and toxicity. Unfortunately, their low concentration (part per trillion) and complexity of matrices (soil, sediments, milk, etc.) generally require analytical methods characterised by several pre-treatment steps and expensive instrumentation. For example, EPA standard procedures [11] involve several clean-up/ pre-concentration steps followed by high resolution gas chromatography coupled to high resolution mass spectrometry (HRGC-HRMS). Soxhlet extraction with toluene, purification on silica gel and alumina packed columns, percolation on activated carbon/celite column are necessary, causing waste of time, use of high quantity of sample and pure solvents, increased costs. New pre-treatment systems have been introduced to improve dioxin analytical determination [10,12,13]. Also in this field, recognition capabilities of MIPs could be beneficial.

Only few works are reported on MIPs against dioxins [1,14,15]. Advances in this field would also reduce in principle the need for high resolution techniques (HRGC-HRMS). In last years alternative methods, applying techniques such as GC-quadrupole ion storage MS in tandem mode (GCQISTMS/MS), fast GC (FGC) or two-dimensional GC (GC $\times$ GC) coupled to time-of-flight MS (TOFMS), were developed to analyse PCDDs and similar compounds (PCDFs, PCBs) [12,16]; they are less sensitive than the reference one but attractive in terms of speed, cost and sample turnover. Nonetheless, reduction of complexity of extract would further reduce resolution request. For example, a resolving power greater than 10,000 is necessary to discriminate between $(\mathrm{M}+2)^{+}$ion from TCDD

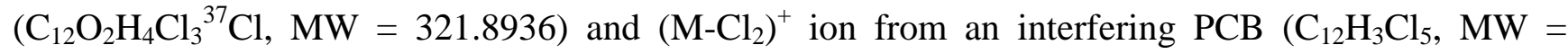
321.86774) in confirmatory procedures for peak identification (see Table 3 in ref.17). A correctly designed MIP for dioxin could separate TCDD from interferent PCBs during pre-treatment steps.

Among MIPs for dioxins, Lübke et al. [1] report the synthesis of a polymer specific for 2,3,7,8tetrachlorodibenzodioxin (TCDD) by covalent imprinting (MIP1). The procedure is very elegant, but it is laborious and time consuming. In the perspective of a wide use of MIP in SPE technology, the noncovalent approach appears more desirable. With this aim, we propose a non-covalent procedure for preparation of a molecularly imprinted polymer for TCDD (MIP2) in which a "dummy” template (2amino-3,7,8-trichlorodibenzodioxin) is employed and the functional co-monomer is 2methacryloyloxybenzoic acid. This scheme represents a significant simplification of the original one presented by Lübke et al.. Influence of template bleeding in dioxin analysis is avoided, since template used in the proposed non-covalent approach is only a close analogue of analyte. 


\section{Experimental Section}

2.1 Materials and Methods: 4-vinylbenzyl chloride, 3,5-dihydroxyanisole, 2,4,5trichloronitrobenzene, hydrazine hydrate, methacrylic anhydride, oxalyl chloride, methacrylic acid, 4chlorocatechol and anhydrous nonane were purchased from Aldrich and used as received. 2,3,7,8tetrachlorodibenzodioxin was purchased from Wir (Italy). Silica gel (Merck) was used for the chromatographic separations. Solvents were dried and distilled under $\mathrm{N}_{2}$ atmosphere. FT-IR spectra were performed on a JASCO FT-IR-430 instrument. GC-MS spectra were recorded on a HewlettPackard GC/Mass MSD 5973 instrument on capillary column SE 30. ${ }^{1} \mathrm{H}$-NMR spectra were recorded on a Bruker $\mathrm{AC}-400$ at room temperature in $\mathrm{CDCl}_{3}$, internally referenced to $\mathrm{CHCl}_{3}$. The centrifugation of polymer suspensions was achieved using a centrifuge Hettich EBA20 before LC-MS analyses, performed using an Agilent HPLC/MS 1100 SL series instrument (mass range 100-3000 a.m.u.) equipped with an Atmospheric Pressure Chemical Ionisation (APCI) interface. The samples, prepared in dry nonane, were introduced in the mass spectrometer employing an autosampler Agilent G1313A with a $1 \%$ accuracy having a syringe with a maximum capacity of $100 \mu$ and internal size diameter of $0.17 \mathrm{~mm}$. Methanol/ $\mathrm{CH}_{2} \mathrm{Cl}_{2}$ 9:1 were used as mobile phase with a flux of $0.5 \mathrm{ml}^{*} \mathrm{~min}^{-1}$ in the following conditions: negative ion mode, drying gas flow $\left(\mathrm{N}_{2}\right) 4 \mathrm{l}^{*} \mathrm{~min}^{-1}$, nebulizer pressure $4 \mathrm{~atm}$, drying gas temperature $350^{\circ} \mathrm{C}$, nebulizer temperature $350{ }^{\circ} \mathrm{C}$, capillary voltage $4000 \mathrm{~V}$ and corona current $10 \mu \mathrm{A}$.

2.2 Synthesis of templates: Fig.1 illustrates the synthesis of template $N$-(2-(3,7,8trichlorodibenzodioxinyl))-2-methacryloyloxybenzamide (6) for MIP1: details on all steps can be found in ref.1. The synthetic scheme of template (compound (3)) for MIP2 consists of only steps for products (1) to (4) in Fig.1.

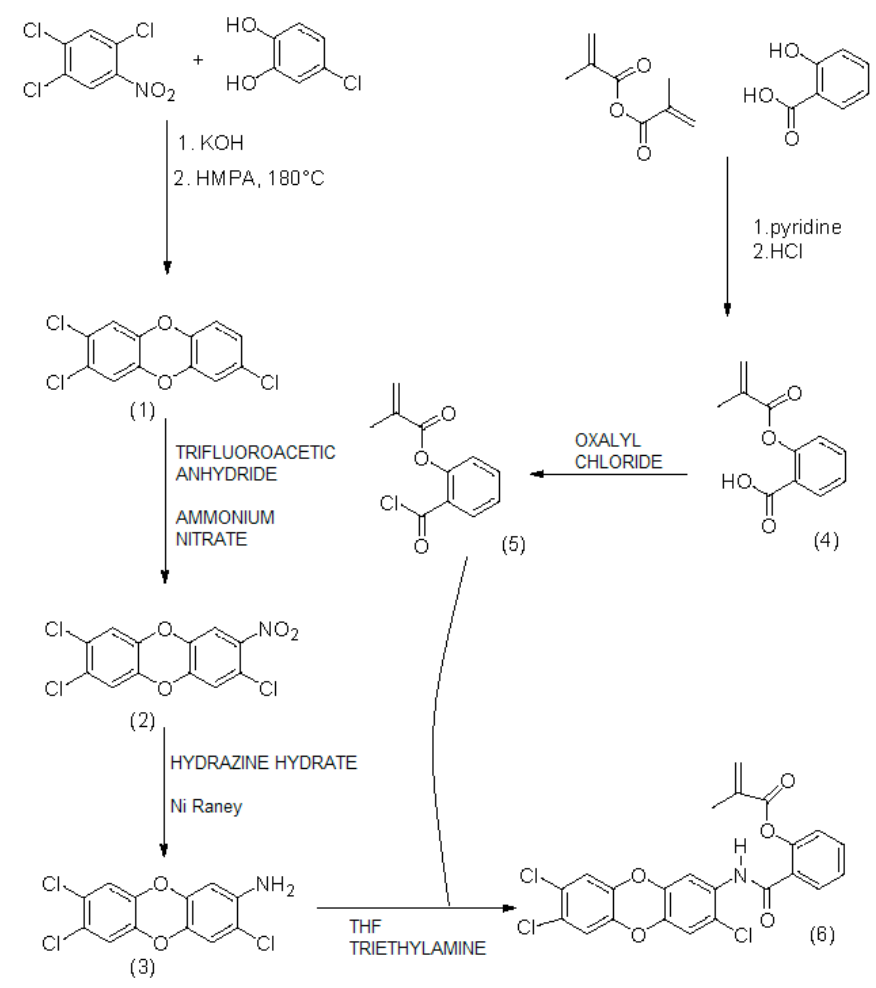

Figure 1. Synthesis of template N-(2-(3,7,8-trichlorodibenzodioxinyl))-2-metachryloiloxybenzamide) for MIP1, (adapted from Lübke et al., 1998). 
Details of syntheses of precursors (1), (2), (5), (6) are not reported since they were performed and verified as in the original paper [1]. Details of syntheses of (3) and (4) are described because they are directly involved in the new polymerisation scheme.

2.3 Synthesis and characterisation of 2-amino-3,7,8-trichloro-dibenzodioxin (3) and 2methacryloyloxybenzoic acid (4): the main passages, found in both schemes, were the reduction of (2) to (3) and the preparation of (4). The former reaction was realised heating at reflux hydrazine hydrate and Nickel Raney in ethanol and tetrahydrofuran (THF), while (2), dissolved in THF, was added slowly. Afterwards a specified amount of hydrazine hydrate was added, then the mixture was cooled and filtered after the reaction was completed. The filtrate was evaporated to give (3) as a white solid with a high yield (96\%). This compound is easily oxidised, thus it must be stored at $4^{\circ} \mathrm{C}$ under nitrogen. It was characterised by GC-MS (m/z 301) (Fig.2).

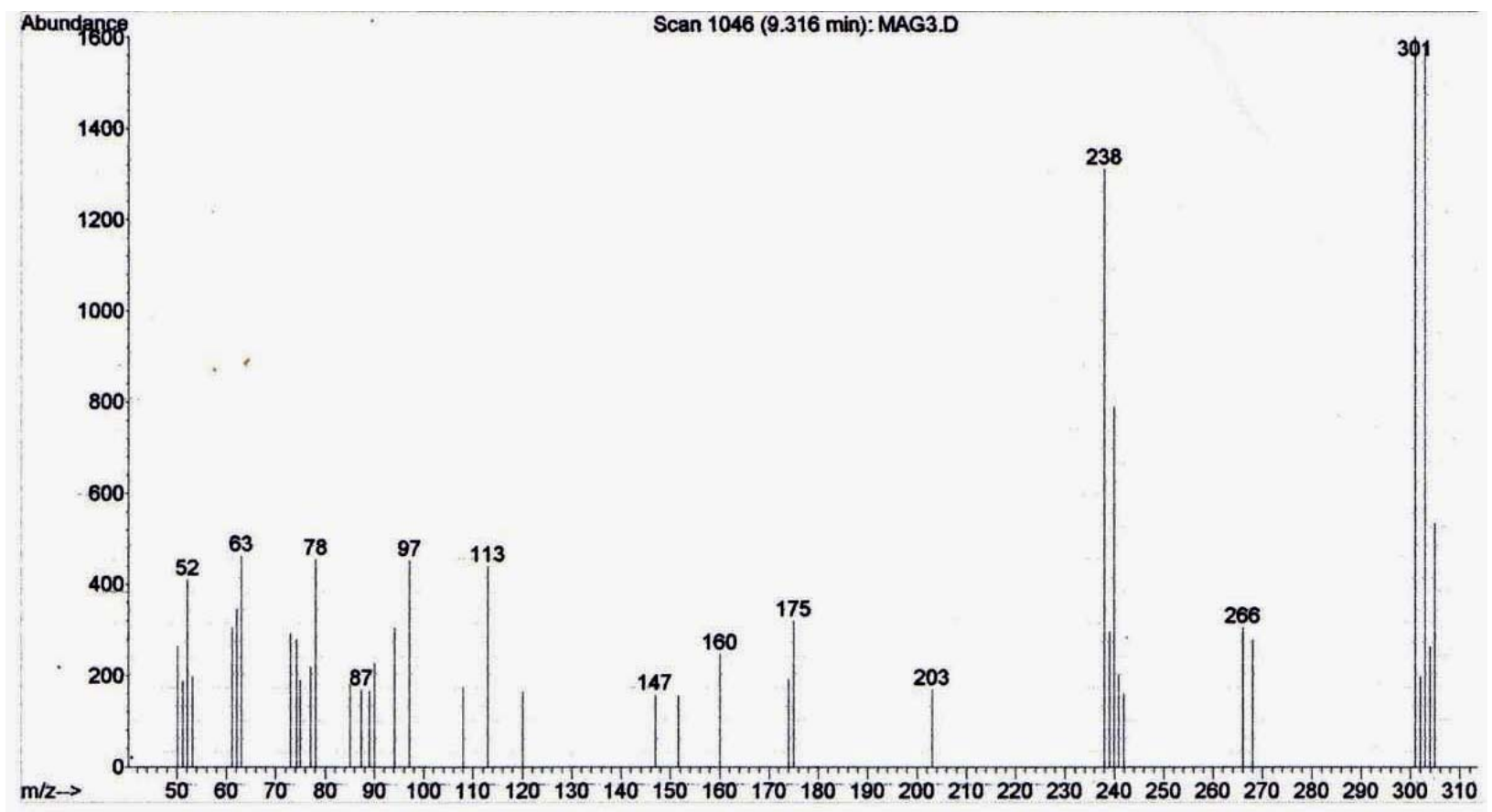

Figure 2. MS spectrum of 2-amino-3,7,8-trichloro-dibenzodioxin.

(4) was produced starting from salicylic acid and methacrylic anhydride. The anhydride was added to the acid solution in pyridine, cooled in ice. The mixture was stirred overnight and then added to dilute $\mathrm{HCl}$ in excess and ice. The product was oily, thus it was extracted with diethyl ether and extracts were dried over $\mathrm{MgSO}_{4}$ and evaporated. Pure compound was obtained by several crystallization steps from hexane, giving colourless crystals with a $42 \%$ yield. Melting point, IR, and ${ }^{1} \mathrm{H}-\mathrm{NMR}$ characterisations were in accordance with literature [18]. In Fig.3 is reported ${ }^{1} \mathrm{H}$-NMR spectrum: $\left(\mathrm{CDCl}_{3}\right) \delta(\mathrm{ppm})$ 7.20-8.20 (m, 4H, aromatic protons), 5.77-6.40 (m, 2H, =C=CH$), 2.07$ (s, 3H, $\left.-\mathrm{CH}_{3}\right)$.

2.4 Polymer synthesis and template removal: Polymerisation and template removal schemes for covalent and non-covalent approach are sketched in Figs. 4 and 5, respectively. (7) was synthesised and characterised as in ref.1. Polymerisation was carried out at $65{ }^{\circ} \mathrm{C}$ in presence of azobisisobutyronitrile (AIBN) as radical initiator and divinylbenzene (DVB) as cross-linking monomer in place of more classical ones because of extreme conditions during template removal (Lübke et al., 
1998). Also template removal was performed as in ref.1. A non imprinted polymer (NIP) was also prepared following the scheme of Fig.4, but using methacrylic acid in place of template.

Details on polymers syntheses are reported in Table 1.

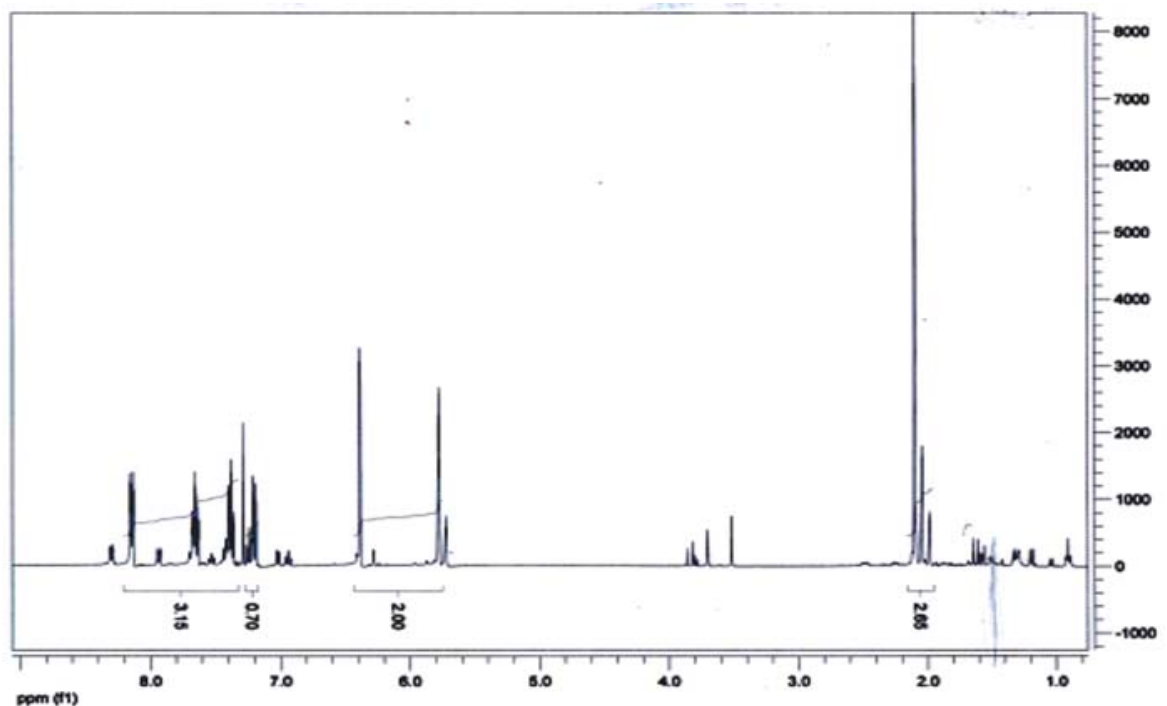

Figure 3. ${ }^{1} \mathrm{H}$-NMR of 2-methacryloyloxybenzoic acid.

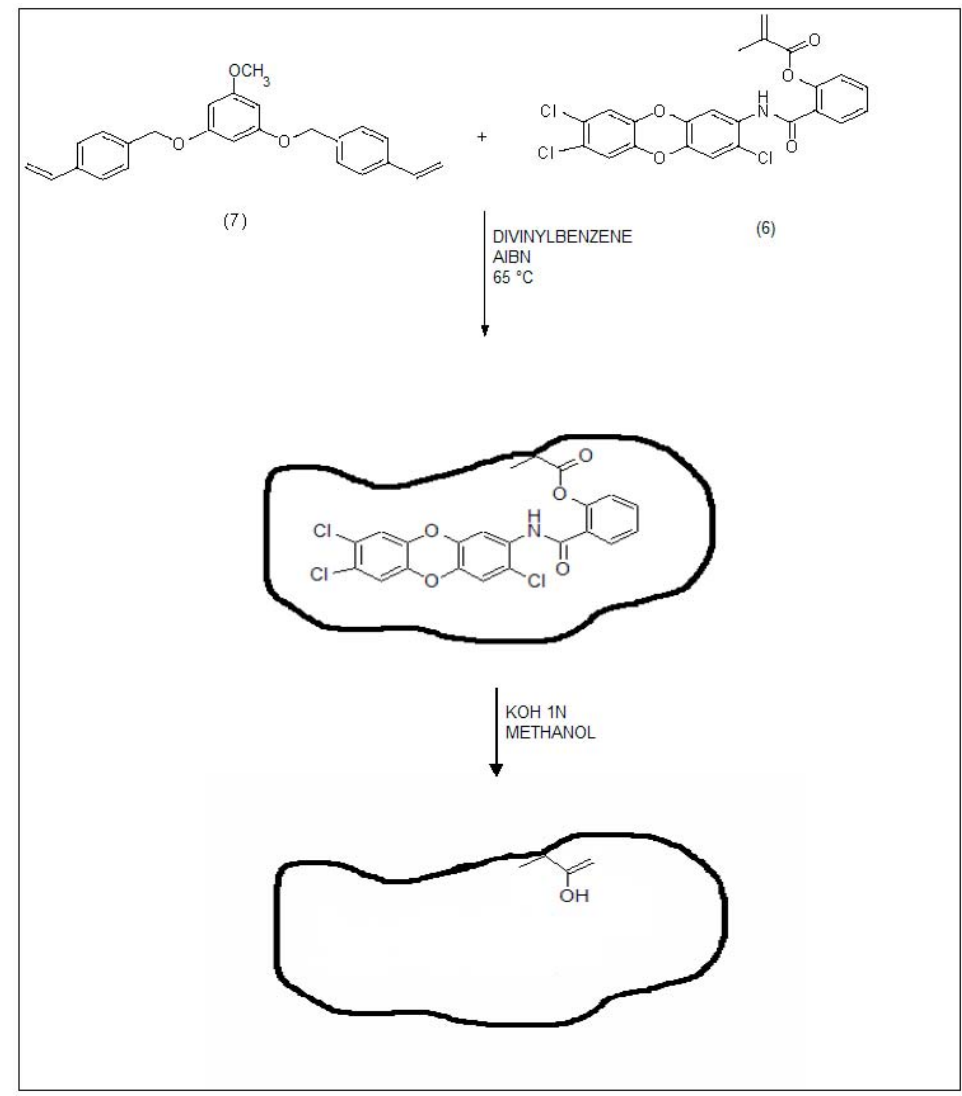

Figure 4. Synthesis of MIP1 and template removal. 


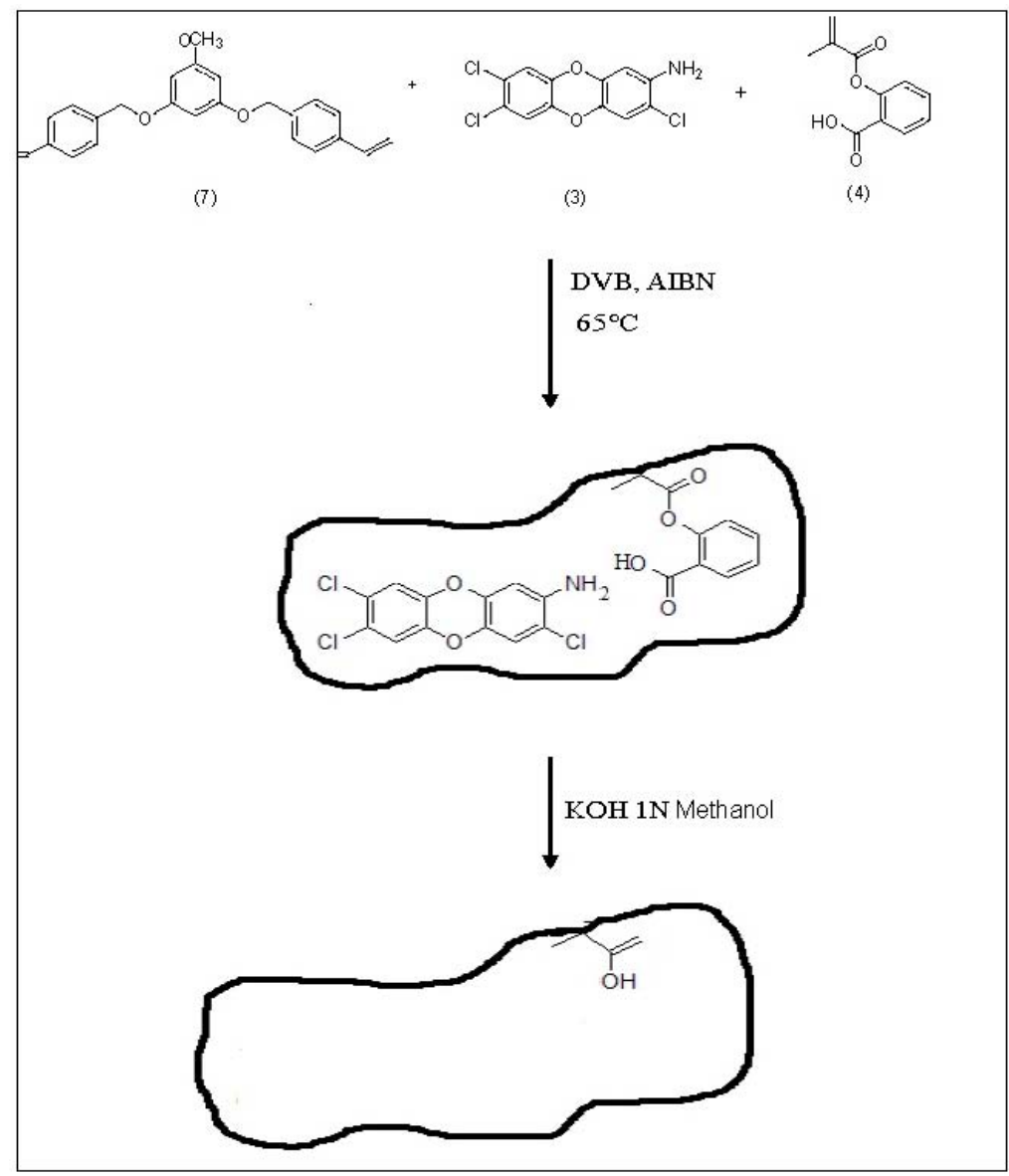

Figure 5. Synthesis of MIP2 and template removal.

Table 1. Experimental details of polymers syntheses (see text).

\begin{tabular}{|c|c|c|c|c|c|c|c|}
\hline Polymer & $\begin{array}{c}\text { DVB / mol } \\
\text { \% }\end{array}$ & $\mathbf{( 3 )} / \mathbf{m o l}$ \% & $\begin{array}{c}\mathbf{( 4 )} / \mathbf{m o l} \\
\mathbf{\%}\end{array}$ & $\begin{array}{c}\mathbf{( 6 )} / \mathbf{m o l} \\
\mathbf{\%}\end{array}$ & $\begin{array}{c}\text { methacrylic } \\
\text { acid / mol \% }\end{array}$ & $\begin{array}{c}\text { (7) / mol } \\
\text { \% }\end{array}$ & Solvent \\
\hline NIP & 94.75 & $/$ & $/$ & $/$ & 0.25 & 5 & THF \\
\hline MIP1 & 94.75 & $/$ & $/$ & 0.25 & $/$ & 5 & THF \\
\hline MIP2 & 94.5 & 0.25 & 0.25 & $/$ & $/$ & 5 & THF \\
\hline
\end{tabular}

The polymers were finely grinded in a mortar and employed as such in template removal and rebinding experiments.

2.5 Re-binding experiments on TCDD: $10.0 \mathrm{mg}$ of single grinded polymers (MIP1, MIP2, NIP) were weighed into screw cap vials and incubated for $24 \mathrm{~h}$ with $1 \mathrm{ml}$ of a $6.96 \mathrm{pM}$ solution of TCDD in anhydrous nonane. After this time contact, solutions were filtered and remained TCDD concentration was determined by APCI-MS. Five standard TCDD solutions (1.39-6.96 pM) were prepared by dilution of the $6.96 \mathrm{pM}$ solution in dry nonane for calibration curve. $50 \mu \mathrm{l}$ of each solution was injected monitoring the negative ion at $\mathrm{m} / \mathrm{z} 319.10$ (in single ion mode) that resulted the more intense 
ion peak obtained for 2,3,7,8-TCDD. The amount of extracted TCDD was calculated by difference in concentration before and after exposure to polymers. Re-binding experiments were performed in triplicate.

\section{Results and Discussion}

Lübke et al. [1] showed how the "sacrificial spacer" methodology [19] could be applied to build a MIP with specific recognition sites for TCDD (MIP1). In fact, the application of a close analogue of dioxin, $N$-(2-(3,7,8-trichlorodibenzodioxinyl))-2-methacryloyloxybenzamide (6), fused with a salicylic acid moiety ensures the introduction of methacrylic acid residue into polymer after hydrolysis for template removal (Fig.4). In this way, dioxin binding was allowed by the enhanced hydrogen bond with chlorine atom as methacrylic acid residue is situated in a fixed position in the active site; it can be noticed that salicylic acid provides an intramolecular hydrogen bonding between oxygen atom and NH group [1], essential for creating right space arrangement in the binding site.

In our strategy, the production of methacrylic functionality under suitable geometric constraints was achieved by a more simplified scheme (see experimental) with less extensive synthetic work (the chlorination of (4) and the production of (6) are avoided).

As it was discussed, the proposed MIPs differ in templates but the monomers employed in the polymerisation step are nearly the same (figs.4-5). In fact, previous study [1] underlined the importance of choosing a suitable co-monomer to improve $\pi$ - $\pi$ interactions with the electro-deficient dioxin system. On the basis of original results, 1-methoxy-3,5-bis(4-vinylbenzyloxy)benzene (7) was selected as a useful second recognition element into the binding sites. Comparison between figures 4 and 5 gives indication on the different imprinting approaches. While template for MIP1 (compound (6)) is covalently bonded to the methacrylic moiety to be polymerised, MIP2 is based on ionic interactions and/or hydrogen bonding between the template (dioxin analogue (3)) and (4) (bearing the methacrylic polymerisable group). The common drawback of non-covalent approach represented by template bleeding in analytical applications of MIPs is overcome in our proposed scheme by the use of a "dummy" template.

To assess imprinting effect and evaluate specific binding capacity of MIP2, re-binding experiments were performed on MIP2 and NIP (Table 2). Results were also compared with performance of polymer MIP1, as evaluated in this work. A concentration of TCDD nearly 3 orders of magnitude lower than the one employed in the original paper was selected for re-binding experiments. This choice was aimed to lower influence of aspecific binding and evidence specific differences among tested polymers.

It should be noted that polymer grain size distribution was not characterised. Even so, results of rebinding experiments were quite reproducible (Table 2).

Data suggest that an imprinting effect is exhibited by both MIPs, higher for MIP1 as it could be expected. However, it is noteworthy that the imprinting effect is remarkably high also for the new proposed MIP; this feature was advantageously coupled to a simplified preparation scheme for MIP precursors. Thus, MIP2 could represent an interesting compromise between an easy polymer preparation and a good selectivity for dioxins. 
Our calculated ratios are significantly higher than in the original paper: we believe that this finding simply indicates that at the higher concentration employed by Lübke et al., aspecific binding has a significant role.

Finally, it should be observed that extracted TCDD is lower in our measurements than in the original paper [1]: this could be tentatively attributed to the lower TCDD concentration and the coarser grain size polymer employed.

Table 2. Results of TCCD re-binding test (triplicate experiments) and comparison with literature report.

\begin{tabular}{|lllll|}
\hline Polymer & Extracted TCDD & Extracted & Imprinting & Imprinting \\
& pmol $^{*} \mathbf{g}^{-1}$ & TCDD $\left(^{*}\right)$ & factor & factor $\left(^{*}\right)$ \\
& & pmol $^{*} \mathbf{g}^{-1}$ & & \\
\hline NIP & $0.098 \pm 0.003$ & $10.78 \pm 0.57$ & $/$ & $/$ \\
\hline MIP1 & $0.54 \pm 0.01$ & $11.98 \pm 0.32$ & MIP1/NIP 5.51 & MIP1/NIP 1.11 \\
\hline MIP2 & $0.239 \pm 0.004$ & $/$ & MIP2/NIP 2.44 & $/$ \\
\hline
\end{tabular}

* Literature report [1]

\section{Conclusions}

A synthesis of a molecularly imprinted polymer for TCDD by a non- covalent approach has been proposed on the basis of a simplification of Lübke et al.'s covalent imprinting scheme and employing a “dummy” template. Re-binding experiments revealed that TCDD binds significantly to the novel polymer, suggesting that non-covalent approach has been successfully applied. Although other work is necessary for real applications, obtained results open the way to the use of the new developed polymer in SPE of dioxins. On this way, optimisation of the synthetic process (which has been tested only under one set of experimental conditions) as well as a more accurate and extensive analytical characterisation of the imprinted polymers are next goals to succeed in pre-treatment of real samples.

\section{Acknowledgements}

The authors acknowledge financial support from MIUR (PRIN2004 - 2004034021_003). We would like to thank Dr. M.J. Whitcombe for his precious advice during the synthesis of precursors of MIP1.

\section{References and Notes}

1. Lübke, M.; Whitcombe, M.J.; Vulfson, E.N. A Novel Approach to the Molecular Imprinting of Polychlorinated Aromatic Compounds. Journal of the American Chemical Society 1998, 120, 13342-13348. 
2. Pichon, V. Environmental sample treatment: evolution and perspectives. Analusis 1997, 25, 101106.

3. Barcelò, D.; Hennion, M.-C. Online sample handling strategies for the trace-level determination of pesticides and their degradation products in environmental waters. Analytica Chimica Acta 1995, 318, 1-41.

4. Hennion, M.-C. Sample handling strategies for the analysis of non-volatile organic compounds from environmental water samples. TrAC, Trends in Analytical Chemistry 1991, 10, 317-323.

5. Mahony, J.O.; Nolan, K.; Smyth, M.R.; Mizaikoff, B. Molecularly imprinted polymers-potential and challenges in analytical chemistry. Analytica Chimica Acta 2005, 534, 31-39.

6. Masquè, N.; Marcè, R.M.; Borrull, F. Molecularly imprinted polymers: new tailor-made materials for selective solid-phase extraction. TrAC, Trends in Analytical Chemistry 2001, 20, 477-486.

7. Lanza, F.; Sellergren, B. The application of molecular imprinting technology to solid phase extraction. Chromatographia 2001, 53, 599-611.

8. Haupt, K. Molecularly Imprinted Polymers: The Next Generation. Analytical Chemistry 2003, 75, 376A-383A.

9. Chapuis, F.; Pichon, V.; Hennion, M.-C. Molecularly Imprinted Polymers: Developments and Applications of New Selective Solid-Phase Extraction Materials. LC-GC Europe 2004, 17, 408417.

10. Focant, J.-F.; Pirard, C.; De Pauw, E. Automated sample preparation-fractionation for the measurement of dioxins and related compounds in biological matrices: a review. Talanta 2004, 63, 1101-1113.

11. USEPA, Method 1613, Revision B, Tetra through Octa-Chlorinated Dioxins and Furans by Isotope Dilution HRGG/HRMS, Office of Water Engineering and Analysis Division (4303) Washington, D.C. 204600, 1994.

12. Djien Liem, A.K. Important developments in methods and techniques for the determination of dioxins and PCBs in foodstuffs and human tissues. TrAC, Trends in Analytical Chemistry 1999, 18, 499-507.

13. Chen, C.-Y.; Hass, J.R.; Albro, P.W. A Screening Method for Polychlorinated Dibenzo-p-dioxins (PCDDs), Dibenzofurans (PCDFs), and Non-Ortho Polychlorinated Biphenyls (PCBs) in Biological Matrixes. Environmental Science \& Technology 2000, 34, 5172-5176.

14. Kobayashi, T.; Reddy, P.S.; Ohta, M.; Abe, M.; Fujii, N. Molecularly Imprinted Polysulfone Membranes Having Acceptor Sites for Donor Dibenzofuran as Novel Membrane Adsorbents: Charge Transfer Interaction as Recognition Origin. Chemistry of Materials 2002, 14, 2499-2505.

15. Hosoya, K.; Watabe, Y.; Ikegami, T.; Tanaka, N.; Kubo, T.; Sano, T.; Kaya, K. A molecular recognition strategy towards tetra-chlorinated dibenzo-p-dioxins, TCDDs. Biosensors and Bioelectronics 2004, 20, 1185-1189.

16. Focant, J.-F.; Pirard; C.; De Pauw, E. Recent advances in mass spectrometric measurement of dioxins. Journal of Chromatography A 2005, 1067, 265-275.

17. Djien Liem, A.K. Basic aspects of methods for the determination of dioxins and PCBs in foodstuffs and human tissue. TrAC, Trends in Analytical Chemistry 1999, 18, 429-439. 
18. Grainger, J.; Liu, Z.; Sirimanne, S.; Francis, V.L.; Patterson, D.G. Jr. Organohalogen Compounds 11(Dioxin '93, 13th International Symposium on Chlorinated Dioxins and Related Compounds, 1993) 1993, pp. 207-210.

19. Whitcombe, M.J.; Rodriguez, M.E.; Villar, P.; Vulfson, E.N. A New Method for the Introduction of Recognition Site Functionality into Polymers Prepared by Molecular Imprinting: Synthesis and Characterization of Polymeric Receptors for Cholesterol. Journal of the American Chemical Society 1995, 117, 7105-7111.

(C) 2006 by MDPI (http://www.mdpi.org). Reproduction is permitted for noncommercial purposes. 